\title{
Interaction network of immune-associated genes affecting the prognosis of patients with glioblastoma
}

\author{
XIAOHONG HOU $^{1 *}$, JIALIN CHEN ${ }^{2 *}$, QIANG ZHANG ${ }^{1}$, YINCHUN FAN ${ }^{1}$, \\ CHENGMING XIANG $^{1}$, GUIYIN ZHOU ${ }^{1}$, FANG CAO $^{1}$ and SHENGTAO YAO ${ }^{1}$ \\ ${ }^{1}$ Department of Cerebrovascular Disease, Affiliated Hospital of Zunyi Medical University; ${ }^{2}$ Department of Neonatology, \\ The First People' s Hospital of Zunyi Affiliated to Zunyi Medical University, Zunyi, Guizhou 563000, P.R. China
}

Received October 15, 2019; Accepted October 6, 2020

DOI: $10.3892 /$ etm.2020.9493

\begin{abstract}
Glioblastoma multiforme (GBM) is a common malignant tumor type of the nervous system. The purpose of the present study was to establish a regulatory network of immune-associated genes affecting the prognosis of patients with GBM. The GSE4290, GSE50161 and GSE2223 datasets from the Gene Expression Omnibus database were screened to identify common differentially expressed genes (co-DEGs). A functional enrichment analysis indicated that the co-DEGs were mainly enriched in cell communication, regulation of enzyme activity, immune response, nervous system, cytokine signaling in immune system and the AKT signaling pathway. The co-DEGs accumulated in immune response were then further investigated. For this, the intersection of those co-DEGs and currently known immune-regulatory genes was obtained and a differential expression analysis of these overlapping immune-associated genes was performed. A risk model was established using immune-regulatory genes that affect the prognosis of patients with GBM. The risk score was significantly associated with the prognosis of patients with GBM and had a significant independent predictive value. The risk model had high accuracy in predicting the prognosis of patients with GBM [area under the receiver operating characteristic curve $(\mathrm{AUC})=0.764$ ], which was higher than that of a previously reported model of prognosis-associated biomarkers (AUC $=0.667$ ). Furthermore, an interaction network was constructed by using immune-regulatory genes and transcription factors affecting the prognosis of patients with GBM and the University of California Santa Cruz database was used to perform a preliminary analysis of the transcription factors
\end{abstract}

Correspondence to: Dr Shengtao Yao, Department of Cerebrovascular Disease, Affiliated Hospital of Zunyi Medical University, 201 Dalian Road, Zunyi, Guizhou 563000, P.R. China

E-mail: yst@zmu.edu.cn

*Contributed equally

Key words: immune gene, poor prognosis, glioblastoma, Gene Expression Omnibus, The Cancer Genome Atlas and immune genes of interest. The interaction network of immune-regulatory genes constructed in the present study enhances the current understanding of mechanisms associated with poor prognosis of patients with GBM. The risk score model established in the present study may be used to evaluate the prognosis of patients with GBM.

\section{Introduction}

Glioblastoma multiforme (GBM) is one of the most malignant tumor types of the central nervous system, with short median survival and poor prognosis. The major treatment is combined therapy, including surgery, radiotherapy and chemotherapy (1-3). In spite of these combinations, the efficacy of GBM treatments remains unsatisfactory, placing a serious burden on society and affected families (4).

In recent years, the significant role of the immune microenvironment in tumors has been increasingly elucidated. The immune microenvironment has an important role in the prognosis of patients with GBM (5-7). In the immune microenvironment of tumors, immune cells and stromal cells have an important role and affect the prognosis of patients (8-13). An algorithm called ESTIMATE was designed to predict immune infiltration by estimating immune cells and stromal cells with specific expression values (14). In addition, transcription factors are able to bind to specific sequences on the 5 ' end of the gene, thereby regulating gene expression $(15,16)$. Transcription factors have a key role in the development of GBM (5,17-19).

The era of excessive data has provided a convenient platform to explore the molecular mechanisms of various diseases. Microarray analysis is of great research and application value and has an important role in the discovery of molecular markers for diagnosis and prognosis, as well as novel drug targets (20). In the present study, to further investigate the association between transcription factors and immune-regulatory genes, data from the Assay for Transposase-Accessible Chromatin (ATAC) database from patients with GBM were analyzed and peak coverage on chromosomes was determined. Finally, an interaction network of the prognosis-associated immune-regulatory genes and transcription factors was established, providing a possible immune gene regulatory network for genes associated with poor prognosis in GBM. 


\section{Materials and methods}

Data sources. Gene Expression Omnibus (GEO) is a public functional genomic database of integrated gene expression data, gene chips and microarrays. The gene expression datasets used in the present study were downloaded from the GEO online platform (https://www.ncbi.nlm.nih.gov/geo/) (21). The selected datasets were GSE2223, including 4 normal glioma samples and 27 GBM samples, GSE50161, including 13 normal samples and 34 GBM samples, and GSE4290, including 23 normal glioma samples and 81 GBM samples. To further verify the association between immune-regulatory genes and GBM, the level-3 gene expression profile and clinical data of patients with GBM were downloaded from The Cancer Genome Atlas (TCGA) database (Data release/version 17.0; https://portal.gdc.cancer.gov/). The immune score and stromal score were determined from data obtained from the database using the ESTIMATE algorithm (14). From the TCGA database, a small number of non-cancerous control tissues from subjects without GBM were available. The most common sites of GBM are the frontal lobe, temporal lobe and parietal lobe. In order to reduce bias, the corresponding 102 normal samples were downloaded from the University of California Santa Cruz (UCSC) database (https://xena.ucsc.edu/) and the data were then corrected using the 'removevBatchEffect' function of the limma package (22). ATAC with high-throughput sequencing (ATAC-seq) data for patients with GBM were downloaded from the ATAC database (https://gdc.cancer. gov/about-data/publications/ATACseq-AWG).

Differentially expressed genes (DEGs). R software (version 3.5.1) was used to analyze the three datasets GSE2223, GSE50161 and GSE4290, as well as the TCGA and GTEX data. Screening criteria were set to $\mathrm{P}<0.05$ and loglfold change (FC) $\mid>1$, and finally, the common DEGs (co-DEGs) of the datasets were obtained.

Retrieval of immune gene and transcription factor data. The enrichment analysis of co-DEGS from the three GEO datasets focused on results associated with immunization. For further study, tumor-associated immune genes and transcription factors were downloaded from the IMMPORT database (https://www.immport.org/) and the Cistrome database (http://cistrome.org/). The DEGs among the downloaded immune genes and transcription factors were obtained using the screening conditions of $\mathrm{P}<0.05$ and $\log \mid \mathrm{FCl}>0.58$ (since there were fewer eligible results when setting $\log \mid \mathrm{FCl}>1$ in the present study, $\log \mid \mathrm{FCl}>0.58$ was used).

Enrichment analysis of co-DEGs. The co-DEGs from the GEO datasets were uploaded to the Funrich software (23) for Gene Ontology and Kyoto Encyclopedia of Genes and Genomes enrichment analysis and the corresponding functional terms and pathways were obtained. $\mathrm{P}<0.05$ was considered to indicate statistical significance.

Immune gene and transcription factor analysis. The intersection of the co-DEGs from GEO, the DEGs from TCGA and the immune-associated genes was used to perform a subsequent differential expression analysis. Pearson correlation analysis between the differentially expressed immune genes and transcription factors with the prognosis of GBM was then performed with a correlation coefficient of the absolute value $>0.4$ and $\mathrm{P}<0.05$ considered to indicate statistical significance.

Construction of Cox risk model. First, the association of immune genes with the prognosis of patients GBM was determined using univariate Cox regression analysis and a total of 17 prognostic genes were obtained. Subsequently, multivariate Cox regression analysis was used to construct a best-fitting prognostic model and a risk score formula was then established by including each of these selected genes [in total, seven key genes were selected according to the minimum criteria, these genes were calcium binding protein a10 (S100A10), mitogen-activated protein kinase 3 (MAPK3), serpin family A member 3 (SERPINA3), Fc fragment of IgG receptor IIb (FCGR2B), endothelin receptor type A (EDNRA) and p21 activated kinase 1 (PAK1) and the patients were divided into high/low risk score groups]. Patients were classified into high- or low risk-groups with the median risk score as the cutoff.

Multivariate analysis. To determine whether the immune risk score independently affects the survival of patients with GBM, univariate and multivariate Cox proportional hazards model analyses of the influence of risk scores and clinical features of patients with GBM on survival were performed.

Interaction network. In the present study, the 'corrplot' package of $\mathrm{R}$ software was used for interaction network correlation analysis and a correlation coefficient $>0.4$ and $\mathrm{P}<0.001$ were considered to indicate a significant correlation. Subsequently, Cytoscape software (24) was used to construct an interaction network for the immune genes associated with the prognosis of patients with GBM, differentially expressed transcription factors and immune cells from the TIMER database (https://cistrome.shinyapps.io/timer/).

ATAC-seq data analysis. ATAC standardized matrix data were downloaded from the ATAC database and through 'TxDb. Hsapiens', 'UCSC.hg38.knownGene, org.Hs.eg.db', 'clusterProfiler', 'karyoploteR' and 'ChIPseeker' package analyses, the distribution of ATAC-seq peaks on chromosomes was determined. To further assess whether Snail family transcriptional repressor 2 (SNAI2)/MYC and C-X-C motif chemokine receptor 4 (CXCR4)/Serpin family A member 3 (SERPINA3) may have binding sites, they were analyzed through the UCSC Genome Browser tool (minimum score $=400$ ).

Correlation analysis between risk score and immune cells. The TCGA expression data of immune cells of patients with GBM were downloaded from the TIMER database. The 'corrplot' package of R software was used to perform a Pearson correlation analysis of risk scores with the immune cells infiltration level.

Statistical analysis. All statistical analyses were performed using R software (v. 3.5.1). An independent-samples t-test was used for comparison between groups. The Cox proportional hazards regression model was used for logistic regression. Missing values from downloaded datasets were filled using multiple imputation methods (25). 


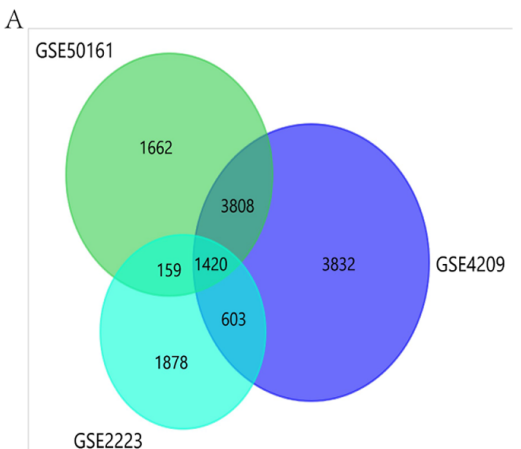

D

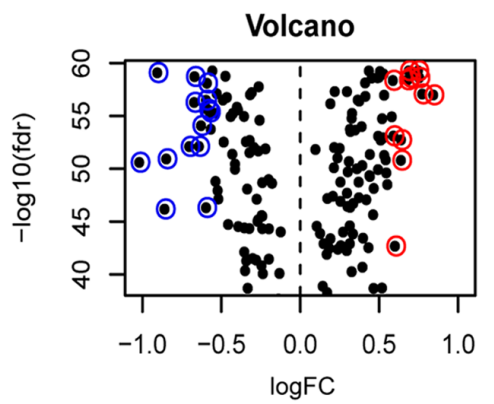

F

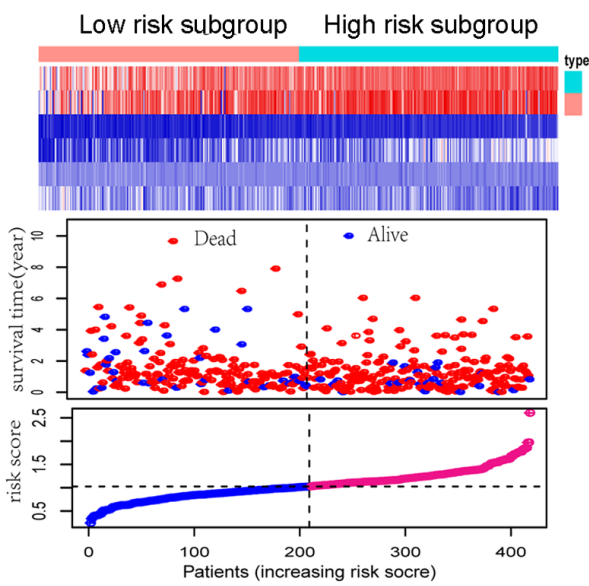

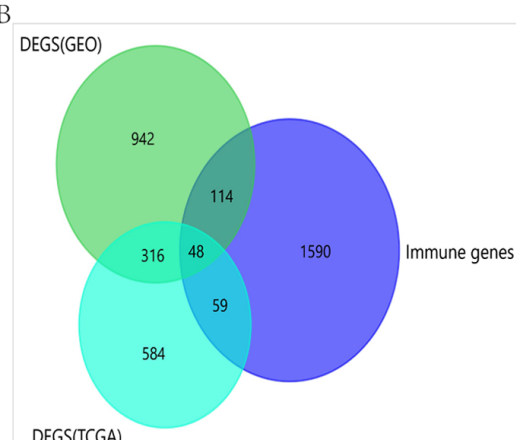

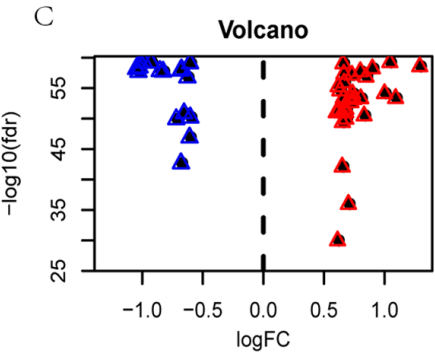

$E$

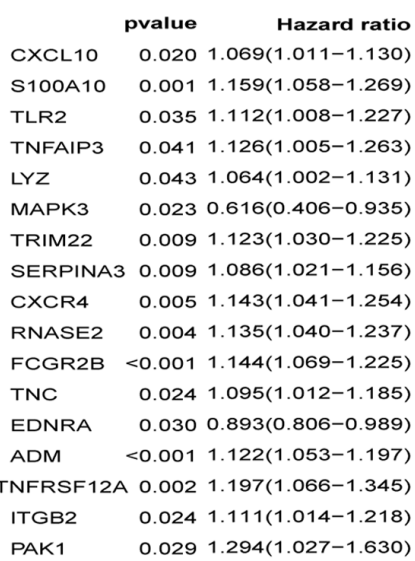

G

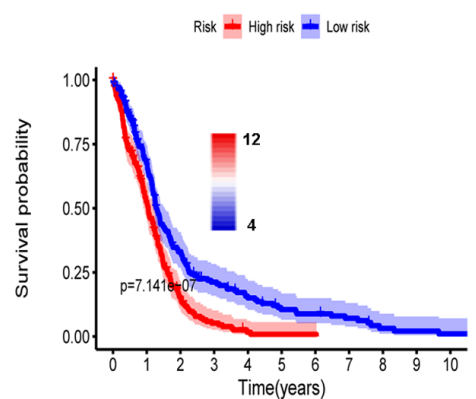

$\mathrm{H}$
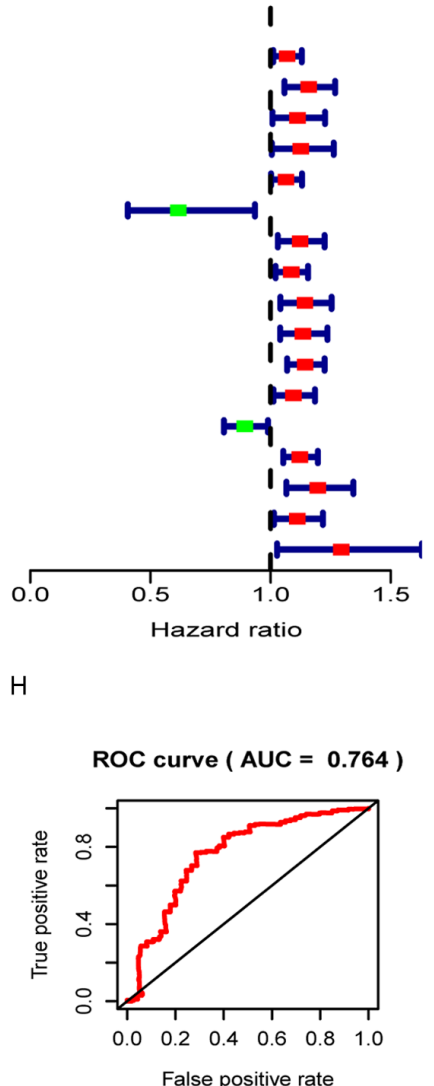

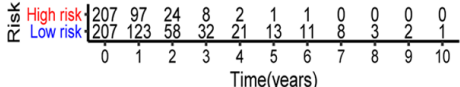

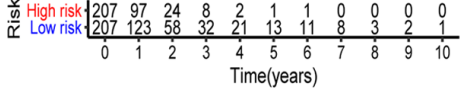

Figure 1. Screening of prognosis-related immune genes and differentially expressed transcription factors and construction of risk model. (A) Venn diagram of common DEGs among the GSE4290, GSE50161 and GSE2223 gene expression datasets. (B) Venn diagram of the intersection of DEGs and known immune-associated genes. (C) Volcano plots displaying differential expression analysis of immune-associated genes and (D) transcription factors. Red indicates high expression and blue indicates low expression. Screening conditions were set to $\mathrm{P}<0.05$ and $\log \mid \mathrm{FCl}>0.58$. (E) Univariate Cox regression analysis identified 17 immune-associated genes affecting the prognosis of patients with GBM and the HR of most genes was $>1$. (F) Risk scoring model for immune gene construction. Red indicates death and blue denotes survival. (G) Impact of high and low risk scores on the prognosis of patients with GBM. A higher risk score indicates a worse prognosis. (H) Receiver operating characteristic curve of the risk scoring model (area under the curve $=0.764$ ). DEG, differentially expressed gene; GEO, gene expression omnibus; GBM, glioblastoma multiforme; TCGA, The Cancer Genome Atlas; FC, fold change; FDR, false discovery rate; HR, hazard ratio; ROC, receiver operating characteristic; AUC, area under the ROC curve; CXCL10, C-X-C Motif Chemokine 10; S100A10, S100 Calcium Binding Protein A10; TLR2, Toll like receptor 2; TNFAIP3, TNF Alpha Induced Protein 3; LYZ, Lysozyme; MAPK3, Mitogen-Activated Protein Kinase 3; TRIM22, Tripartite Motif Containing 22; SERPINA3, Serpin family A member 3; CXCR4, C-X-C motif chemokine receptor 4; RNASE2, Ribonuclease A Family Member 2; FCGR2B, Fc fragment of IgG receptor IIb; TNC, Tenascin C; EDNRA, Endothelin Receptor Type A; ADM, Adrenomedullin; TNFRSF12A, TNF Receptor Superfamily Member 12A; ITGB2, Integrin Subunit Beta 2; PAK1, P21 (RAC1) Activated Kinase 1.

\section{Results}

Identification of DEGs. The datasets from the GSE2223, GSE50161 and GSE4290 gene chips were processed using
$\mathrm{R}$ software. The screening conditions were set to $\log \mid \mathrm{FCl}>1$ and $\mathrm{P}<0.05$, and 1,420 co-DEGs were obtained (Fig. 1A). Furthermore, 48 immune-associated genes were obtained after the DEGs and the immune-associated genes were taken 


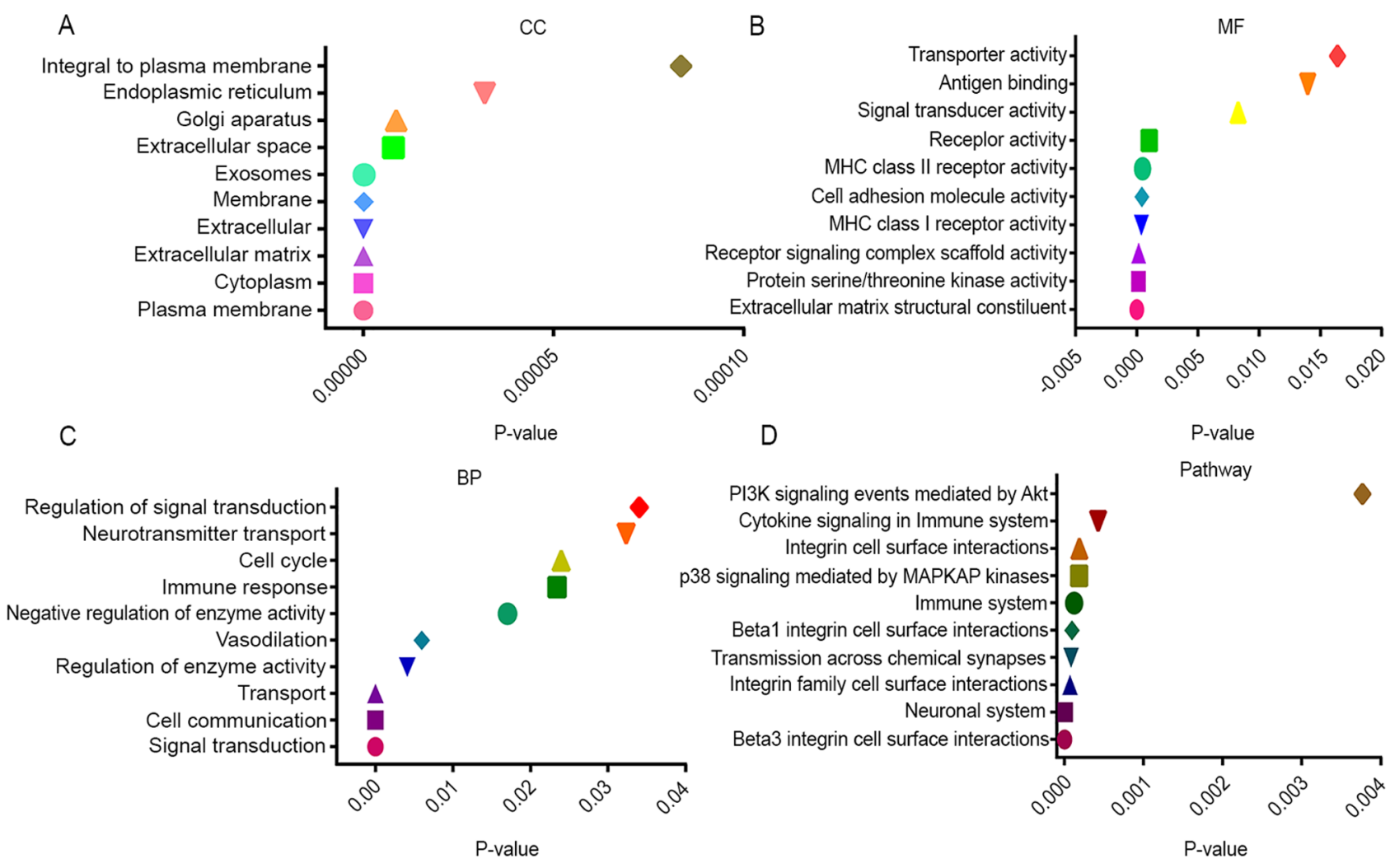

Figure 2. Enrichment analysis of co-DEGs in the Gene Expression Omnibus datasets. Gene Ontology terms in the categories (A) CC, (B) MF and (C) BP. (D) Kyoto Encyclopedia of Genes and Genomes pathways. The results indicated that the co-DEGs were accumulated in the immune microenvironment and the AKT signaling pathway. co-DEG, common differentially expressed gene; CC, cellular component; MF, molecular function; BP, biological process; MHC, major histocompatibility complex; MAPKAP, MAP kinase-activated protein kinase.

together (Fig. 1B). The differentially expressed immune-associated genes and transcription factors (27 differentially expressed transcription factors) are presented in Fig. 1C and D.

Enrichment analysis. Enrichment analysis of co-DEGs indicated that the major functional terms and pathways were cell communication, regulation of enzyme activity, immune response, nervous system, p38 signaling mediated by mitogen-activated protein kinase-activated protein kinase (MAPKAP) kinases, cytokine signaling in immune system and PI3K signaling events mediated by AKT (Fig. 2). Gene set variation (GSVA) enrichment analysis of CXCR4 and SERPINA3 indicated a significant positive correlation with AKT signaling pathways, which were mutually verified in the TCGA and Chinese Glioma Genome Atlas (CGGA) databases (http://www.cgga.org.cn/; Fig. S1).

Evaluation of risk models. In total, 14 immune genes associated with GBM prognosis were obtained by univariate COX regression analysis (Fig. 1E), A risk-score formula was created based on the expression values of these six key genes $($ Fig. $1 \mathrm{~F})$, as follows: Risk score $=(0.08 \times$ expression value of S100A10) + (-0.466 x expression value of MAPK3) $+(0.095 \mathrm{x}$ expression value of SERPINA3) $+(0.071 \mathrm{x}$ expression value of FCGR2B $)+(-0.154 \mathrm{x}$ expression value of EDNRA $)+(0.253 \times$ expression value of PAK1). Finally, the patients were divided into high/low risk groups. The prognosis of the two groups was significantly different, where the high risk group was worse than the low risk group (Fig. 1G). The risk model constructed from prognosis-associated immune-regulatory genes had high accuracy in predicting the prognosis of patients with GBM (AUC=0.764) (Fig. 1H), which was higher than that of a previously reported model constructed from prognosis-associated biomarkers (AUC=0.667; Fig. S2) (10).

Interaction network. In the present study, Cytoscape software was used to construct an interaction network for the immune-regulatory genes (associated with the prognosis of GBM), transcription factors and immune cells (Fig. 3A). The circles indicate the immune-regulatory genes, the diamonds indicate the transcription factors and the squares indicate the immune cells. Red nodes indicate high expression and blue indicates low expression. Red lines indicate positive regulation and blue lines indicate negative regulation. There were 41 nodes in the network, where CXCR4 and SERPINA3 were found to be the key core node genes. Survival analysis identified four transcription factors linked to immune-regulatory genes that affected the prognosis of GBM, namely BRF1, MYC, SNAI2 and SOX4 (Fig. 3B-E, respectively).

Clinical data of patients with GBM. From the GBM patient data that were downloaded (Table I), patients with no survival information were removed and the 417 remaining patients were analyzed. The cohort comprised 248 male and 169 female patients with an age range of 10-89 years, an immune score of $-1,448$ to 3,210 and a stromal score of $-3,055$ to 2,016 . The classification was divided into four subtypes as follows: Classical (30.7\%), mesenchymal (29.3\%), neural (15.3\%) 
A

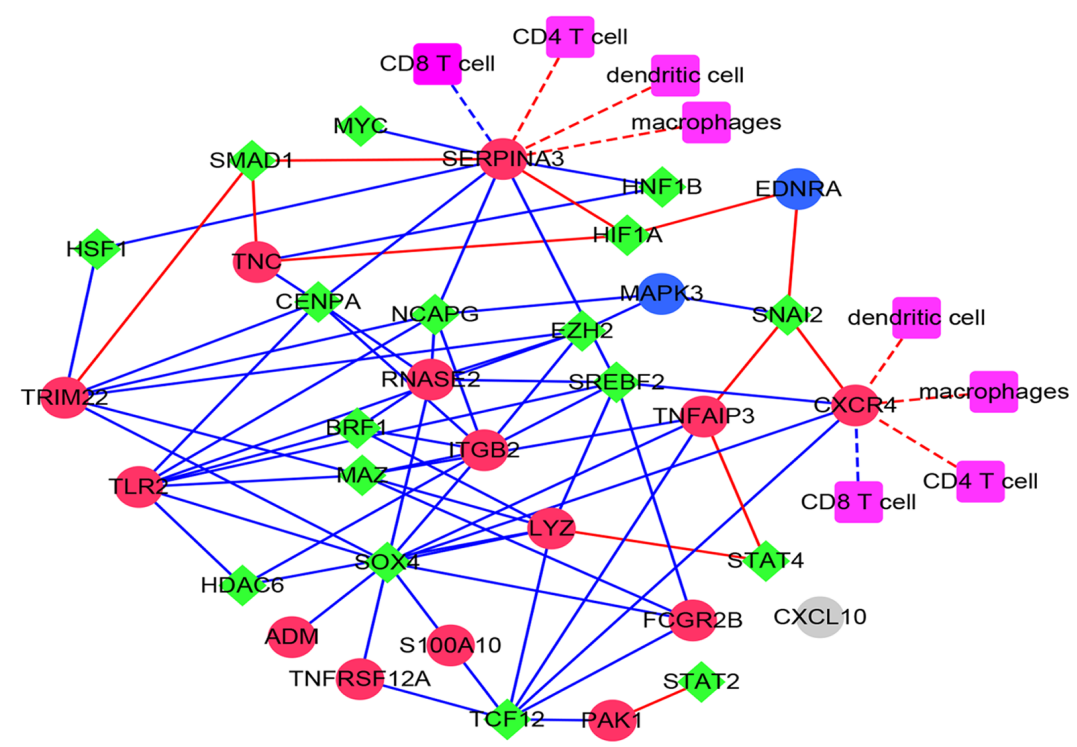

B

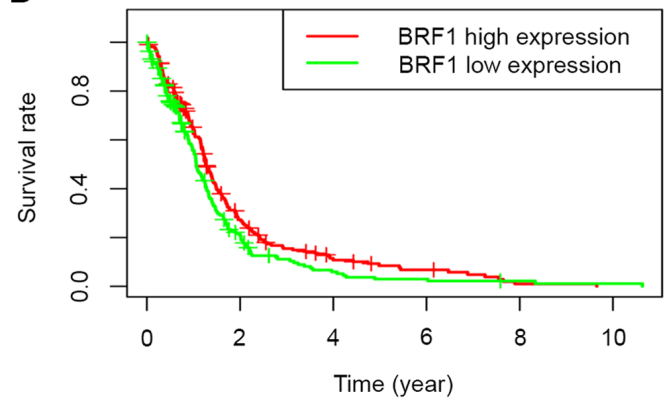

D

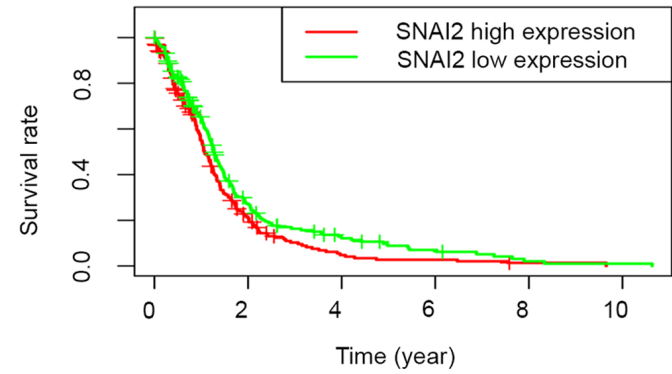

C

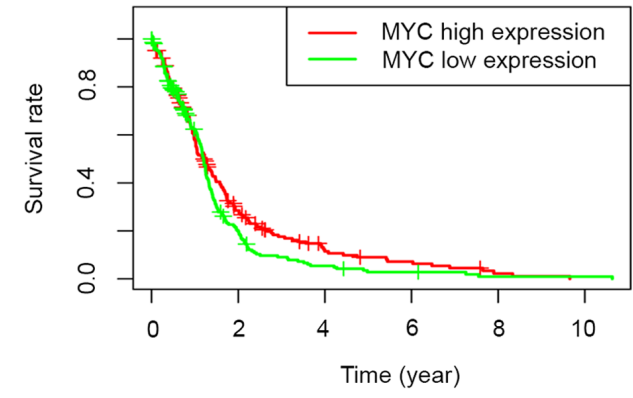

$E$

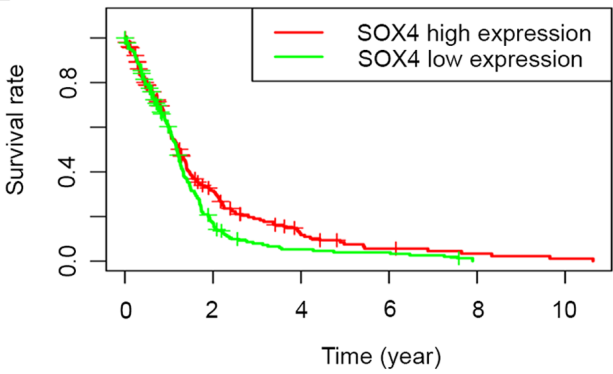

Figure 3. Search for transcription factors related to immune genes and prognosis, and construction of interaction network. (A) Interaction network of prognosis-associated immune genes, transcription factors and immune cells. The circles indicate the immune genes, the diamonds indicate transcription factors, red nodes indicate high expression and blue nodes indicate low expression, red lines indicate positive regulation, blue lines indicate negative regulation and gray indicates no obvious correlation; red/blue dashed lines indicate possible positive/negative effects. (B-E) Survival analysis identified four transcription factors linked to immune genes affecting the prognosis of glioblastoma multiforme. (B) There were BRF1, (C) MYC, (D) SNAI2 and (E) SOX4. BRF1, BRF1 RNA polymerase III transcription initiation factor subunit; MYC, MYC proto-oncogene, BHLH transcription factor; SNAI2, Snail family transcriptional repressor 2, SOX4, SRY-Box Transcription Factor 4.

and proneural (24.7\%). Among the patients, 272 received chemotherapy and $140 \mathrm{did}$ not; furthermore, 365 received radiotherapy and 52 did not. The median follow-up duration was 12.8 months (range, 0-129.4 months).

Analysis of the association between risk score and characteristics of patients with GBM. The association between the risk score and the characteristics of patients with GBM is presented in Fig. 4A-F. The risk score was significantly associated with the prognosis, stromal score and immune score of patients with GBM. Multivariate analysis indicated that the risk score was an independent predictive factor of poor prognosis in patients with GBM (Fig. 4B). High risk scores predicted adverse outcomes in patients with GBM. The immune score and stromal score were significantly associated with the prognosis of patients with GBM (Fig. 4E and F).

ATAC data analysis and search for upstream transcription factors of SERPINA3 and CXCR4. Analysis of the ATAC data indicated that, in addition to the $\mathrm{Y}$ chromosome, there was a 
Table I. The Cancer Genome Atlas Glioblastoma patient characteristics.

\begin{tabular}{lcc}
\hline Clinical characteristics & Total $(\mathrm{n}=417)$ & $\%$ \\
\hline Age $(10-89$ years $)$ & & \\
$<75$ years & 366 & 87.8 \\
$\geq 75$ years & 51 & 12.2 \\
Sex & & \\
Male & 248 & 59.5 \\
Female & 169 & 40.5 \\
Immune score & & \\
$-1,448-3,210$ & 417 & 100 \\
Stromal score & & \\
$-3,055-2,016$ & 417 & 100 \\
Radiotherapy & & \\
Yes & 365 & 87.5 \\
No & 52 & 12.5 \\
Chemotherapy & & \\
Yes & 272 & 66 \\
Yes & 140 & 34 \\
Subtype & & \\
Classical & 128 & 30.7 \\
Mesenchymal & 122 & 29.3 \\
Neural & 64 & 15.3 \\
Proneural & 103 & 24.7 \\
\hline
\end{tabular}

large number of peaks in other chromosomes. Further analysis suggested that the most binding sites were situated near the promoter $(\leq 1 \mathrm{~kb})$, and in the heatmap, a large number of peaks were enriched near the transcription start site (TSS), while the peak enrichment gradually decreased with the distance from the TSS. At the same time, most peaks existed in multiple regions, consistent with previous studies (Fig. 4G-I). In a further search for possible upstream transcription factors of SERPINA3 and CXCR4, possible transcription factors were predicted using the UCSC database, and MYC and SNAI2 were identified, which were also associated with the prognosis of patients with GBM. Further analysis indicated that the promoter regions of SERPINA3 had prospective binding peak sites for MYC and CXCR4 had prospective binding peak sites for SNAI2 (Fig. 5).

Correlation analysis between risk score and immune cells. The 'corrplot' package of R software was used to determine the correlation of the risk scores with immune cells. The results indicated that the risk score was positively correlated with CD4 T cells, dendritic cells, neutrophil cells and macrophages and negatively correlated with CD8 T cells, but the correlation with B-cells did not reach statistical significance (Fig. 6).

\section{Discussion}

In the present study, data of patients with GBM were downloaded from the GEO, TCGA, TIMER, IMMPORT and
CISTROME databases. Co-DEGs were mainly enriched in cell communication, regulation of enzyme activity, immune response, nervous system, p38 signaling mediated by MAPKAP kinases, cytokine signaling in the immune system and PI3K signaling events mediated by AKT. The further GSVA enrichment analysis of CXCR4 and SERPINA3 indicated a positive association with macrophage activation, differentiation and regulation of the AKT signaling pathway.

The present study focused on the tumor immune microenvironment. In the immune microenvironment, immune cells and stromal cells have a key role and affect the prognosis of patients with cancer (14). In the present study, the risk scores were different between subgroups of patients with high and low immune scores and stromal scores, and the risk score was able to be used as an independent risk factor for the prognosis of patients with GBM with statistical significance, and immune scores and stromal scores also affected the prognosis of GBM, which was consistent with previous studies $(8,9)$. In the present study, a total of 48 immune-associated genes were used to perform a prognostic analysis of differentially expressed immune-regulatory genes, yielding 17 genes significantly associated with the prognosis of patients with GBM. The risk model constructed from prognosis-associated immune-regulatory genes had high accuracy in predicting the prognosis of patients with GBM (AUC $=0.764$ ), which was higher than that of a previously reported model constructed from prognosis-associated biomarkers (AUC=0.667) (10). This result suggested that the immune genes screened in the present study were able to better reflect the prognosis of patients with GBM and patients with a high-risk score had a worse prognosis than those with a lower risk score. For further investigation, an interaction network was constructed from prognosis-associated immune genes, differentially expressed transcription factors and immune cells. At the same time, differentially expressed transcription factors were used to predict the prognosis of patients with GBM, indicating that BRF1, MYC, SNAI2 and SOX4 affected the survival of patients with GBM. Combined with the results of the analysis of the UCSC database, SNAI2 and MYC appeared promising, and it was predicted that SNAI2 is able to positively regulate the immune genes CXCR4, and that MYC is able to positively regulate the immune genes SERPINA3. CXCR4 is a CXC chemokine receptor specific for stromal cell-derived factor-1; the protein has 7 transmembrane regions and is located on the cell surface (26). SNAI2 encodes a member of the Snail family of $\mathrm{C} 2 \mathrm{H} 2$-type zinc finger transcription factors (27). Previous studies indicated that CXCR4 affects the proliferation, invasion and angiogenesis of glioma cells by regulating the AKT signaling pathway (28-30), which was consistent with the results of the present enrichment analysis. SNAI2 may be used as a GBM marker, which participates in the epithelial-to-mesenchymal transition and thus affects drug resistance $(31,32)$; it may also enhance the development of tumors by affecting the AKT signaling pathway (32-34). However, whether SNAI2 is able to regulate CXCR4 expression, affect the ATK signaling pathway and thus affect the phenotype of glioma remains to be elucidated. The protein encoded by SERPINA3 is a plasma protease inhibitor and member of the serine protease inhibitor class. Serine protease has an important role in the development of glioma, which may promote the migration and invasion of 
A

\begin{tabular}{|c|c|c|}
\hline \multirow[t]{2}{*}{ A } & & inivariate \\
\hline & pvalue & Hazard ratio \\
\hline Stromal_score & 0.027 & $1.272(1.028-1.574)$ \\
\hline Immune_score & 0.004 & $1.502(1.138-1.982)$ \\
\hline subtype & 0.044 & $1.110(1.003-1.228)$ \\
\hline Age & $<0.001$ & $3.007(2.156-4.194)$ \\
\hline chemo_therapy & $<0.001$ & $2.176(1.651-2.868)$ \\
\hline gender & 0.278 & $0.888(0.717-1.100)$ \\
\hline radiation_therapy & $<0.001$ & $5.817(4.225-8.009)$ \\
\hline riskScore & $<0.001$ & $2.290(1.703-3.080)$ \\
\hline
\end{tabular}

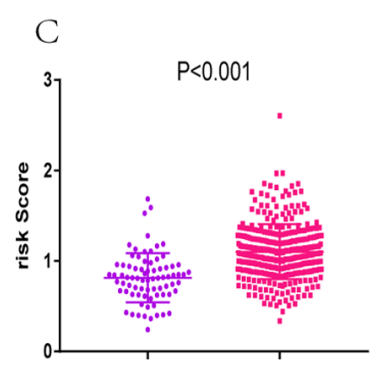

\section{D}

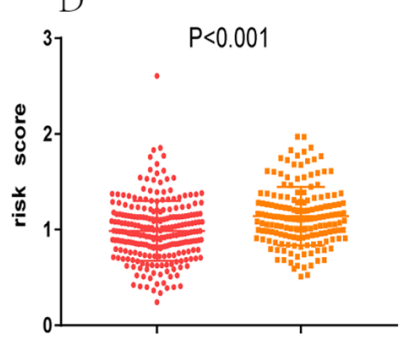

Low immune score High immune score Low stromal score High stromal score G
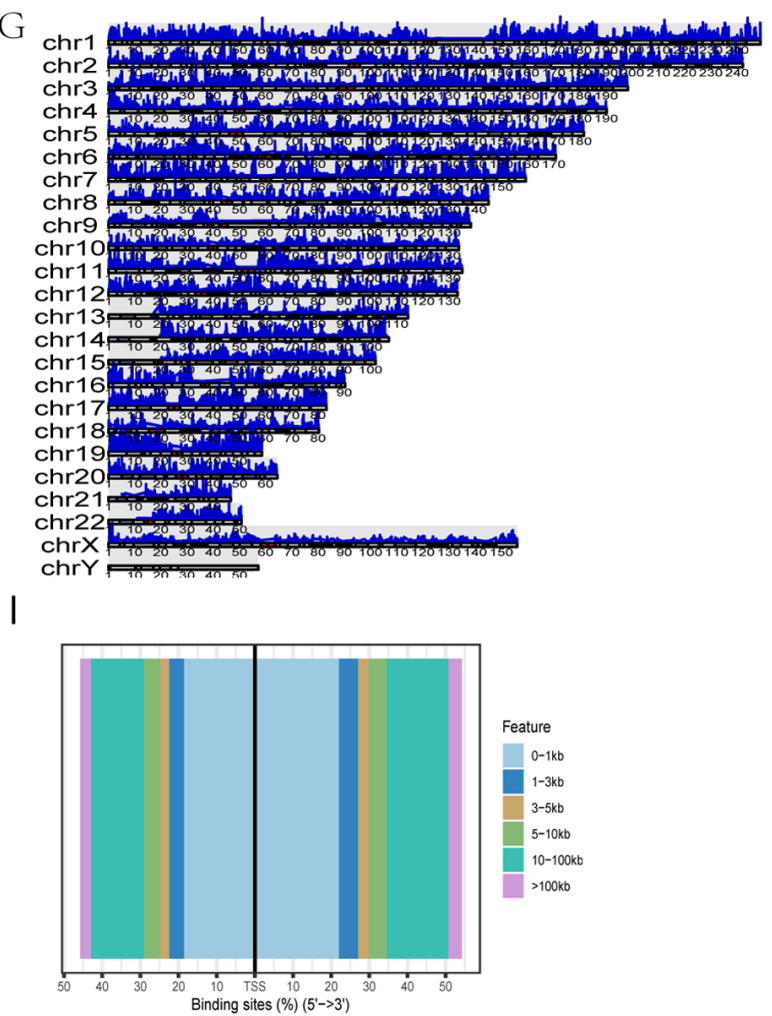

B

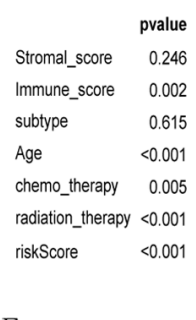

E

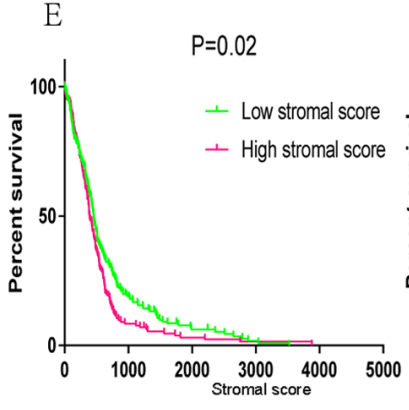

multivariate analysis
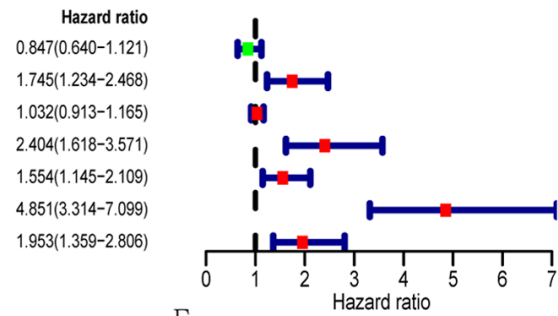

F

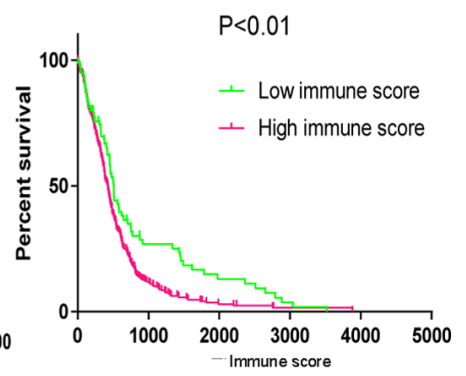

$\mathrm{H}$

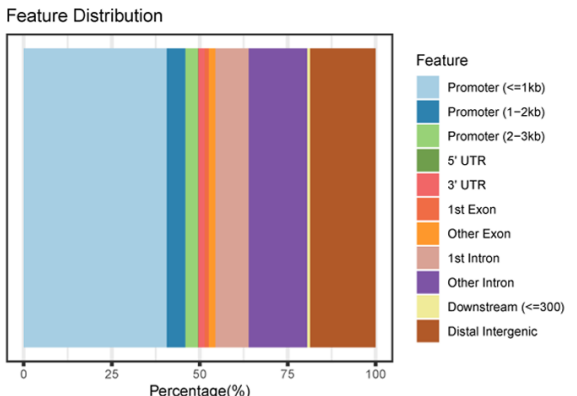

Figure 4. Analysis of clinical characteristics and transcription factor binding sites of GBM. (A) Univariate and (B) multivariate Cox regression analysis of risk scores. The results suggested that the risk score was of independent prognostic value for patients with GBM. (C) High immune and (D) high stromal scores were associated with a higher risk for patients with GBM. The immune score, stromal score and age were stratified into high and low groups using the median value as a cutoff. (E and F) Survival analysis of patients with GBM stratified by (E) the immune score and (F) stromal score. (G) Analysis of data from the Assay for Transposase-Accessible Chromatin database indicated that, in addition to the Y chromosome, there were a large number of peak sites in other chromosomes. (H) Further analysis indicated that the most binding sites were situated near the promoter (I) between 0-1 kbp, a large number of peaks were enriched near the TSS, with gradually less enrichment of peaks further away from the TSS. P $<0.05$ was considered to indicate statistical significance. TSS, transcription start site; GBM, glioblastoma multiforme; UTR, untranslated region; Chr, chromosome.

glioma, but serine protease inhibitor has the opposite role (35). The present study indicated that circulating SERPINA3 may be a marker in GBM and promote the invasion of glioblastic stem cells $(36,37)$, and a positive correlation with the AKT signaling pathway was identified in the GSVA enrichment analysis. However, the specific mechanisms remain elusive. MYC is a proto-oncogene and encodes a nuclear phosphoprotein that has a role in cell cycle progression, apoptosis and cellular transformation of glioma $(38,39)$. A negative regulatory association between MYC and SERPINA3 was indicated in the regulatory network generated in the present study. In GBM, MYC is mainly highly expressed, and it may therefore be speculated that high expression of MYC promotes the development of GBM through inhibition of SERPINA3, 
A

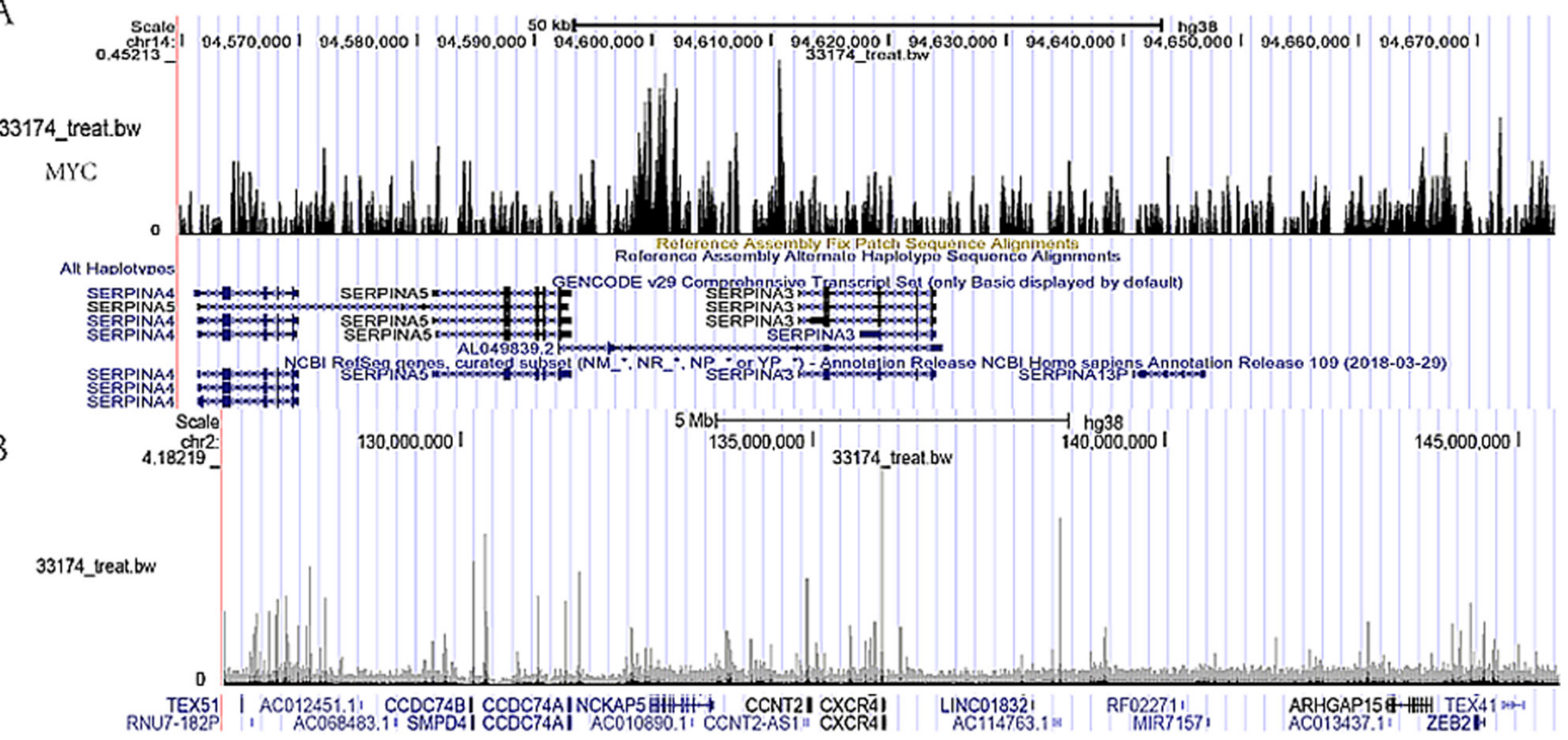

Figure 5. Prediction of binding sites of transcription factors and mRNA in UCSC database. (A) Analysis of the University of California Santa Cruz database revealed SNAI2 peak binding peaks in the promoter regions of the sense sequence in CXCR4 and (B) MYC peak binding peaks in the promoter regions of the sense sequence in SERPINA3, suggesting binding sites between them. CXCR4, C-X-C motif chemokine receptor 4; SERPINA3, Serpin family A member 3; SNAI2, Snail family transcriptional repressor 2.

A

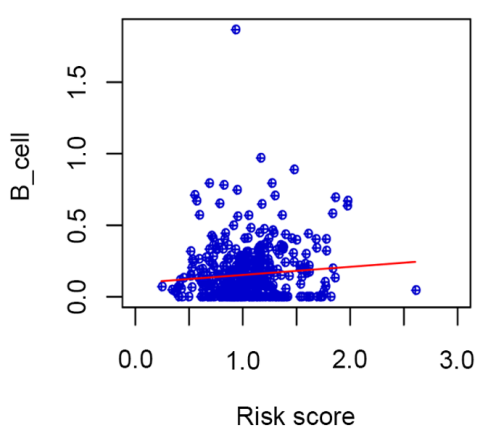

D

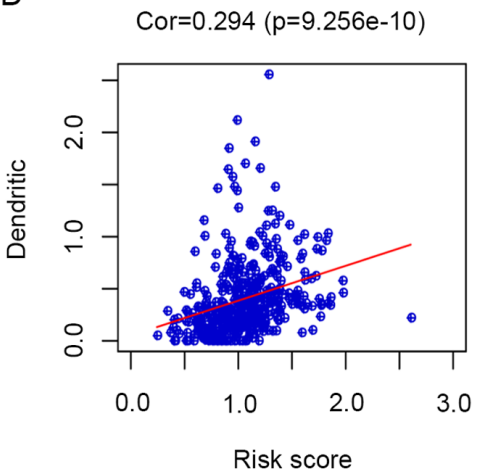

B

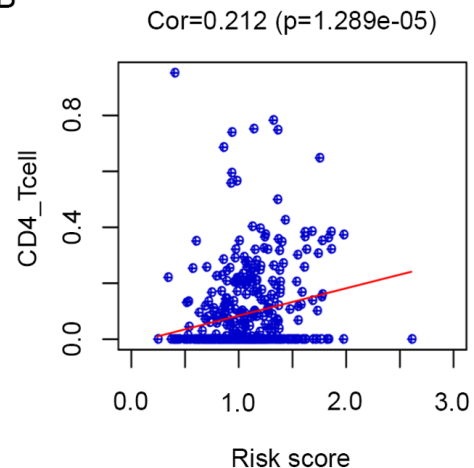

$\mathrm{E}$

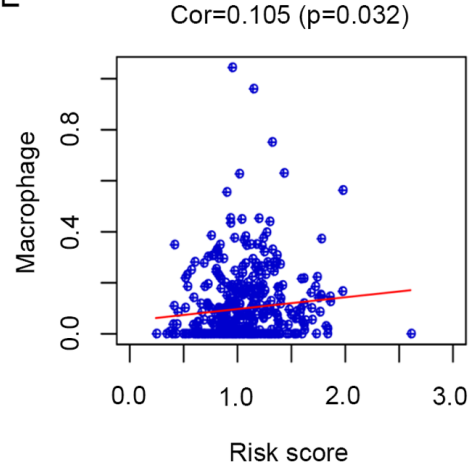

C

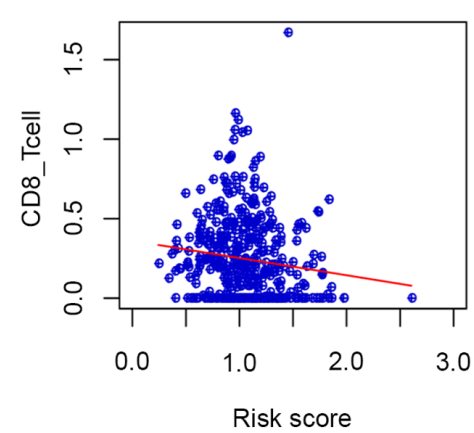

$\mathrm{F}$

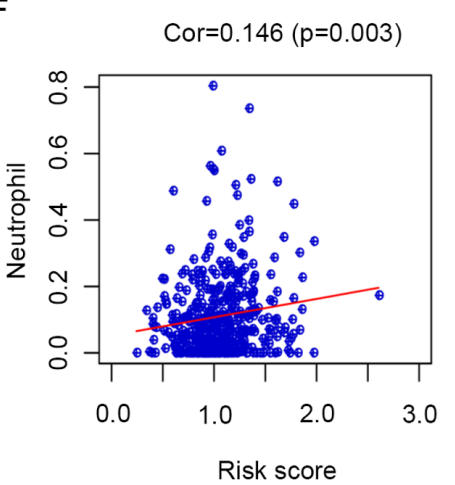

Figure 6. Correlation analysis between risk score and immune cells. The results suggested that the risk score was positively correlated with (A) B cells, (B) CD4 T cells, (D) dendritic cells, (E) macrophages and (F) neutrophils, and (C) negatively correlated with CD8 T cells ( $\mathrm{P}<0.05)$. Cor, correlation coefficient.

which requires further verification. The present study aimed to explore whether binding sites exist between SNAI2 and CXCR4, and between MYC and SERPINA3. The results indicated that there was a large number of peaks on chromosomes of patients with GBM, suggesting that there were numerous transcription factor binding sites on the chromosome and most binding sites were located near the promoter $(\leq 1 \mathrm{~kb})$. The UCSC database was used for analysis, suggesting that the 
CXCR4 and SERPINA3 gene sequences had peaks near the promoter of the transcription factor SNAI2 and MYC, respectively, indicating that there were binding sites between them.

Finally, the association between the risk score and immune cells was analyzed, revealing that the risk score was linked to the immune microenvironment and that the risk score was positively correlated with CD4 T cells and negatively correlated with CD8 T cells. This suggests that a high-risk score may be associated with a secondary elevation of CD4 T cells, dendritic cells and macrophages, as well as a secondary reduction of CD8 T cells. Previous studies have indicated that CD8 T cells and dendritic cells have a positive role in the development of a normal body (40-44). This may indicate that an increase in the risk score may cause a secondary decrease in CD8 T cells, thereby promoting tumor development. It may be hypothesized that high expression of immune-regulatory genes, including SERPINA3 and CXCR4, increased the risk score of patients with GBM, which led to poor prognosis, as well as secondary changes in immune cells in the immune microenvironment, including a secondary decline of CD8 T cells. However, this requires to be confirmed by future studies.

In conclusion, the immune gene interaction network constructed in the present study helps to understand the mechanisms associated with poor prognosis of patients with GBM. A risk scoring system was established in the present study, and high-risk scores indicated poor prognosis of patients with GBM and may be used as an independent risk factor for assessing the prognosis of patients with GBM.

\section{Acknowledgements}

Not applicable.

\section{Funding}

The present study was funded by the Natural Science Foundation of China (grant no. 81660421), The First People's Hospital of Zunyi Joint Research and Development of Science and Technology projects [grant nos. (2010)66 and (2011)24].

\section{Availability of data and materials}

The data that support the findings of this study are openly available in TCGA at http://www.tcga.org/, CGGA at http://www. cgga.org.cn/, TIMER at https://cistrome.shinyapps.io/timer/, UCSC at https://xenabrowser.net/datapages/ and GEO at https://www.ncbi.nlm.nih.gov/geo/.

\section{Authors' contributions}

$\mathrm{XH}, \mathrm{JC}$ and SY conceived the current study, participated in design and coordination, performed the bioinformatic analysis and drafted the manuscript. XH, QZ, and FC participated in the design of the study and performed statistical analysis. YF, CX and GZ participated in the acquisition of data and analysis. All authors read and approved the final manuscript.

\section{Ethics approval and consent to participate}

Not applicable.

\section{Patient consent for publication}

Not applicable.

\section{Competing interests}

The authors declare that they have no competing interests.

\section{References}

1. Louis DN, Ohgaki H, Wiestler OD, Cavenee WK, Burger PC, Jouvet A, Scheithauer BW and Kleihues P: The 2007 WHO classification of tumours of the central nervous system. Acta Neuropathol 114: 97-109, 2007.

2. Dubrow R, Darefsky AS, Jacobs DI, Park LS, Rose MG, Laurans MS and King JT Jr: Time trends in glioblastoma multiforme survival: The role of temozolomide. Neuro-oncol 15: 1750-1761, 2013.

3. Stupp R, Hegi ME, Mason WP, van den Bent MJ, Taphoorn MJ, Janzer RC, Ludwin SK, Allgeier A, Fisher B, Belanger K, et al; European Organisation for Research and Treatment of Cancer Brain Tumour and Radiation Oncology Groups; National Cancer Institute of Canada Clinical Trials Group: Effects of radiotherapy with concomitant and adjuvant temozolomide versus radiotherapy alone on survival in glioblastoma in a randomised phase III study: 5-year analysis of the EORTC-NCIC trial. Lancet Oncol 10: 459-466, 2009.

4. Alexiou GA, Tsiouris S, Kyritsis AP, Voulgaris S, Argyropoulou MI and Fotopoulos AD: Glioma recurrence versus radiation necrosis: Accuracy of current imaging modalities. J Neurooncol 95: 1-11, 2009.

5. Cooper LA, Gutman DA, Chisolm C, Appin C, Kong J, Rong Y, Kurc T, Van Meir EG, Saltz JH, Moreno CS, et al: The tumor microenvironment strongly impacts master transcriptional regulators and gene expression class of glioblastoma. Am J Pathol 180: 2108-2119, 2012.

6. Galon J, Pagès F, Marincola FM, Thurin M, Trinchieri G, Fox BA, Gajewski TF and Ascierto PA: The immune score as a new possible approach for the classification of cancer. J Transl Med 10: 1, 2012.

7. Şenbabaoğlu Y, Gejman RS, Winer AG, Liu M, Van Allen EM, de Velasco G, Miao D, Ostrovnaya I, Drill E, Luna A, et al: Tumor immune microenvironment characterization in clear cell renal cell carcinoma identifies prognostic and immunotherapeutically relevant messenger RNA signatures. Genome Biol 17: 231, 2016.

8. Chang N, Ahn SH, Kong DS, Lee HW and Nam DH: The role of STAT3 in glioblastoma progression through dual influences on tumor cells and the immune microenvironment. Mol Cell Endocrinol 451: 53-65, 2017.

9. Zhang B, Shen R, Cheng S and Feng L: Immune microenvironments differ in immune characteristics and outcome of glioblastoma multiforme. Cancer Med 8: 2897-2907, 2019.

10. Jia D, Li S, Li D, Xue H, Yang D and Liu Y: Mining TCGA database for genes of prognostic value in glioblastoma microenvironment. Aging (Albany NY) 10: 592-605, 2018.

11. Akimoto K, Kimura K, Nagano M, Takano S, To'a Salazar G, Yamashita T and Ohneda O: Umbilical cord blood-derived mesenchymal stem cells inhibit, but adipose tissue-derived mesenchymal stem cells promote, glioblastoma multiforme proliferation. Stem Cells Dev 22: 1370-1386, 2013.

12. Boonstra MC, Verbeek FP, Mazar AP, Prevoo HA, Kuppen PJ, van de Velde CJ, Vahrmeijer AL and Sier CF: Expression of uPAR in tumor-associated stromal cells is associated with colorectal cancer patient prognosis: A TMA study. BMC Cancer 14: 269, 2014.

13. Miki Y, Yashiro M, Okuno T, Kuroda K, Togano S, Hirakawa K and Ohira M: Clinico-pathological significance of exosome marker CD63 expression on cancer cells and stromal cells in gastric cancer. PLoS One 13: e0202956, 2018.

14. Yoshihara K, Shahmoradgoli M, Martínez E, Vegesna R, Kim H, Torres-Garcia W, Treviño V, Shen H, Laird PW, Levine DA, et al: Inferring tumour purity and stromal and immune cell admixture from expression data. Nat Commun 4: 2612, 2013.

15. Lambert SA, Jolma A, Campitelli LF, Das PK, Yin Y, Albu M, Chen X, Taipale J, Hughes TR and Weirauch MT: The Human Transcription Factors. Cell 172: 650-665, 2018. 
16. Malysheva V, Mendoza-Parra MA, Saleem MA and Gronemeyer H: Reconstruction of gene regulatory networks reveals chromatin remodelers and key transcription factors in tumorigenesis. Genome Med 8: 57, 2016.

17. Wang Q, Hu B, Hu X, Kim H, Squatrito M, Scarpace L, deCarvalho AC, Lyu S, Li P, Li Y, et al: Tumor Evolution of Glioma-Intrinsic Gene Expression Subtypes Associates with Immunological Changes in the Microenvironment. Cancer Cell 32: 42-56.e6, 2017.

18. Chong YK, Sandanaraj E, Koh LW, Thangaveloo M, Tan MS, Koh GR, Toh TB, Lim GG, Holbrook JD, Kon OL, et al: ST3GAL1-Associated Transcriptomic Program in Glioblastoma Tumor Growth, Invasion, and Prognosis. J Natl Cancer Inst 108: djv326, 2015

19. Yan ZH, Bao ZS, Yan W, Liu YW, Zhang CB, Wang HJ, Feng Y, Wang YZ, Zhang W, You G, et al: Upregulation of DLX2 confers a poor prognosis in glioblastoma patients by inducing a proliferative phenotype. Curr Mol Med 13: 438-445, 2013.

20. Wang S, Liu F, Wang Y, Fan W, Zhao H, Liu L, Cen C, Jiang X, Sun M and Han P: Integrated analysis of 34 microarray datasets reveals CBX3 as a diagnostic and prognostic biomarker in glioblastoma. J Transl Med 17: 179, 2019.

21. Barrett T, Wilhite SE, Ledoux P, Evangelista C, Kim IF, Tomashevsky M, Marshall KA, Phillippy KH, Sherman PM, Holko M, et al: NCBI GEO: Archive for functional genomics data sets - update. Nucleic Acids Res 41D: D991-D995, 2013.

22. Gentleman R, Carey VJ, Huber W, Irizarry RA and Dudoit S: limma: Linear models for microarray data. In: Bioinformatics and Computational Biology Solutions Using R and Bioconductor. Gentleman R, Carey VJ, Huber W, Irizarry RA and Dudoit S (eds). Springer, New York, NY, pp397-420, 2005.

23. Pathan M, Keerthikumar S, Ang CS, Gangoda L, Quek CY, Williamson NA, Mouradov D, Sieber OM, Simpson RJ, Salim A, et al: FunRich: An open access standalone functional enrichment and interaction network analysis tool. Proteomics 15: 2597-2601, 2015.

24. Shannon P, Markiel A, Ozier O, Baliga NS, Wang JT, Ramage D, Amin N, Schwikowski B and Ideker T: Cytoscape: A software environment for integrated models of biomolecular interaction networks. Genome Res 13: 2498-2504, 2003

25. Lee KJ and Simpson JA: Introduction to multiple imputation for dealing with missing data. Respirology 19: 162-167, 2014

26. Rummel PC, Thiele S, Hansen LS, Petersen TP, Sparre-Ulrich AH, Ulven T and Rosenkilde MM: Extracellular disulfide bridges serve different purposes in two homologous chemokine receptors, CCR1 and CCR5. Mol Pharmacol 84: 335-345, 2013.

27. Cobaleda C,Pérez-Caro M,Vicente-Dueñas C and Sánchez-García I: Function of the zinc-finger transcription factor SNAI2 in cancer and development. Annu Rev Genet 41: 41-61, 2007.

28. Yi L, Zhou X, Li T, Liu P, Hai L, Tong L, Ma H, Tao Z, Xie Y, Zhang C, et al: Notch1 signaling pathway promotes invasion, self-renewal and growth of glioma initiating cells via modulating chemokine system CXCL12/CXCR4. J Exp Clin Cancer Res 38 : 339, 2019.

29. Ping YF, Yao XH, Jiang JY, Zhao LT, Yu SC, Jiang T, Lin MC, Chen JH, Wang B, Zhang R, et al: The chemokine CXCL12 and its receptor CXCR4 promote glioma stem cell-mediated VEGF production and tumour angiogenesis via $\mathrm{PI} 3 \mathrm{~K} / \mathrm{AKT}$ signalling. J Pathol 224: 344-354, 2011.

30. Liu Y, Wei S, Zou Q and Luo Y: Stachydrine suppresses viability \& migration of astrocytoma cells via CXCR4/ERK \& CXCR4/Akt pathway activity. Future Oncol 14: 1443-1459, 2018.

31. Cheng Q, Huang C, Cao H, Lin J, Gong X, Li J, Chen Y, Tian Z, Fang Z and Huang J: A Novel Prognostic Signature of Transcription Factors for the Prediction in Patients With GBM Front Genet 10: 906, 2019.
32. Liao H, Bai Y, Qiu S, Zheng L, Huang L, Liu T, Wang X, Liu Y, $\mathrm{Xu}$ N, Yan X, et al: $\mathrm{miR}-203$ downregulation is responsible for chemoresistance in human glioblastoma by promoting epithelial-mesenchymal transition via SNAI2. Oncotarget 6: 8914-8928, 2015.

33. Fenouille N, Tichet M, Dufies M, Pottier A, Mogha A, Soo JK, Rocchi S, Mallavialle A, Galibert MD, Khammari A, et al: The epithelial-mesenchymal transition (EMT) regulatory factor SLUG (SNAI2) is a downstream target of SPARC and AKT in promoting melanoma cell invasion. PLoS One 7: e40378, 2012.

34. Laakkonen JP, Lappalainen JP, Theelen TL, Toivanen PI, Nieminen T, Jauhiainen S, Kaikkonen MU, Sluimer JC and Ylä-Herttuala S: Differential regulation of angiogenic cellular processes and claudin-5 by histamine and VEGF via PI3K-signaling, transcription factor SNAI2 and interleukin-8. Angiogenesis 20: 109-124, 2017.

35. Huasong G, Zongmei D, Jianfeng H, Xiaojun Q, Jun G, Sun G, Donglin W and Jianhong Z: Serine protease inhibitor (SERPIN) B1 suppresses cell migration and invasion in glioma cells. Brain Res 1600: 59-69, 2015.

36. Miyauchi E, Furuta T, Ohtsuki S, Tachikawa M, Uchida Y, Sabit H, Obuchi W, Baba T, Watanabe M, Terasaki T, et al: Identification of blood biomarkers in glioblastoma by SWATH mass spectrometry and quantitative targeted absolute proteomics. PLoS One 13: e0193799, 2018.

37. Li Y, Dong X, Cai J, Yin S, Sun Y, Yang D and Jiang C: SERPINA3 induced by astroglia/microglia co-culture facilitates glioblastoma stem-like cell invasion. Oncol Lett 15: 285-291, 2018.

38. Ding Z, Liu X, Liu Y, Zhang J, Huang X, Yang X, Yao L, Cui G and Wang D: Expression of far upstream element (FUSE) binding protein 1 in human glioma is correlated with c-Myc and cell proliferation. Mol Carcinog 54: 405-415, 2015

39. Chen H, Guo Y, Sun J, Dong J, Bao Q, Zhang X and Fu F: Preferential Expression of B7-H6 in Glioma Stem-Like Cells Enhances Tumor Cell Proliferation via the c-Myc/RNMT Axis. J Immunol Res 2020: 2328675, 2020.

40. Eiraku Y, Terunuma H, Yagi M, Deng X, Nicol AJ and Nieda M: Dendritic cells cross-talk with tumour antigen-specific CD8 ${ }^{+}$ $\mathrm{T}$ cells, $\mathrm{V} \gamma 9 \gamma \delta \mathrm{T}$ cells and V $\alpha 24 \mathrm{NKT}$ cells in patients with glioblastoma multiforme and in healthy donors. Clin Exp Immunol 194: 54-66, 2018.

41. Prasad S, Gaedicke S, Machein M, Mittler G, Braun F, Hettich M, Firat E, Klingner K, Schüler J, Wider D, et al: Effective Eradication of Glioblastoma Stem Cells by Local Application of an AC133/CD133-Specific T-cell-Engaging Antibody and CD8 T Cells. Cancer Res 75: 2166-2176, 2015.

42. Xu X, Stockhammer F, Schmitt A, Casalegno-Garduno R, Enders A, Mani J, Classen CF, Linnebacher M, Freund M and Schmitt M: Therapeutical doses of temozolomide do not impair the function of dendritic cells and $\mathrm{CD}^{+} \mathrm{T}$ cells. Int $\mathrm{J}$ Oncol 40: 764-772, 2012

43. Pellegatta S, Poliani PL, Stucchi E, Corno D, Colombo CA, Orzan F, Ravanini M and Finocchiaro G: Intra-tumoral dendritic cells increase efficacy of peripheral vaccination by modulation of glioma microenvironment. Neuro-oncol 12: 377-388, 2010.

44. Candolfi M, King GD, Yagiz K, Curtin JF, Mineharu Y, Muhammad AK, Foulad D, Kroeger KM, Barnett N, Josien R, et al: Plasmacytoid dendritic cells in the tumor microenvironment: Immune targets for glioma therapeutics. Neoplasia 14: 757-770, 2012.

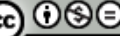

This work is licensed under a Creative Commons Attribution-NonCommercial-NoDerivatives 4.0 International (CC BY-NC-ND 4.0) License. 